\title{
A Product for Special Classes of Monogenic Functions and Tensors
}

\author{
F. Sommen and P. Van Lancker
}

\begin{abstract}
In this paper we define a product for monogenic functions derived from Fischer's decomposition. Although this product can be defined for all monogenic polynomials, it can be canonically defined only for a special subclass of polynomials. The definition of the product can be generalized to the setting of monogenic tensors.
\end{abstract}

Keywords: Clifford algebra, tensors, Clifford analysis, differential forms

AMS subject classification: 15 A 66, 15 A 69, 30 G 35, 58 A 10

\section{Introduction}

Let $R_{m}$ be the Clifford algebra generated by the basis $\left\{e_{1}, \ldots, e_{m}\right\}$ of $R^{m}$ and the defining relations $e_{i} e_{j}+e_{j} e_{i}=-2 \delta_{i j}$. Then the $R_{m}$-valued solutions $f(x)$ of $\partial_{x} f(x)=$ 0 , with $\partial_{x}=\sum e_{j} \partial_{x_{j}}$ being the Dirac operator, are called left monogenic functions. The same name is used for null-solutions of the operator $\partial_{x_{0}}+\partial_{x}$ which is also called generalized Cauchy Riemann operator since it generalizes the Cauchy-Riemann operator in the plane. This is true except for the important fact that the product $f\left(x_{0}, x\right) g\left(x_{0}, x\right)$ of two left monogenic functions is no longer left monogenic. So the question is : Do there exist "product-like operations" for monogenic functions? One such operation is the socalled Cauchy-Kowalewski product (see $[1,5]$ ) which is based on the uniqueness of the left monogenic extension $f\left(x_{0}, x\right)$ of a given real analytic function $f(x)=f(0, x)$. The idea works as follows: Given two left monogenic functions $f$ and $g$, consider the product of real analytic functions $f(0, x) g(0, x)$ and define the Cauchy-Kowalewski product to be the monogenic extension $f \cdot g$ of this. Although well defined, the Cauchy-Kowalewski product is very hard to work with and, except for strip domains, there are no examples of domains for which the space of monogenic functions becomes an algebra. Moreover, the Cauchy-Kowalewski product is quite unnatural to be defined for all monogenic functions. Indeed, the space of left monogenic functions is a right $R_{m}$-module which means that one has to consider Cauchy- Kowalewski extensions of products of restrictions of monogenic functions of the form $\left(f(0, x) e_{1}\right) g(0, x)=f(0, x)\left(e_{1} g(0, x)\right)$, but the function $e_{1} g\left(x_{0}, x\right)$ is usually not left monogenic. Hence, in general there is conceptionally something wrong with this product which again does not mean that it is entirely meaningless; it may be

F. Sommen: University of Gent, Dept. Math. Analysis, Galglaan 2, B - 9000 Gent; Senior Research Associate, NFWO, Belgium

P. Van Lancker: University of Gent, Dept. Math. Analysis, Galglaan 2, B - 9000 Gent 
an interesting and naturally defined product structure for special types of monogenic functions. Moreover, the Cauchy-Kowalewski theorem may be formulated for general analytic surfaces (see, e.g., [6]) which means that a Cauchy-Kowalewski product may be defined with respect to general analytic surfaces such as the unit sphere.

But there is still another theorem that may give rise to a meaningful product, namely the Fischer decomposition of homogeneous polynomials which can be obtained for general differential operators such as the Laplacian or the Dirac operator (see, e.g., [2]). For the Laplacian we have a Fischer decomposition of the form

$$
R_{k}(x)=\sum_{s}|x|^{2 s} H_{k-2 s}(x)
$$

whereby $R_{k}$ is a given homogeneous polynomial of degree $k$ and $H_{k-2 s}$ are uniquely determined spherical harmonics of degree $k-2 s$. This theorem leads to the following well known product structure defined for spherical harmonics (see also [12]) :

Given two spherical harmonics $H_{k}$ and $H_{l}$ one may form the usual product $H_{k} H_{l}$ being a homogeneous polynomial $R_{k+l}$ of degree $k+l$ and then define the harmonic product $H_{k} \circ H_{l}$ to be the highest piece in the Fischer decomposition of $R_{k+l}$.

In Section 1 of this paper we define a Fischer product for spherical monogenics of a special type. This restriction again has to do with the incompatibility of the Fischer product and the right module structure of monogenic functions. But the algebra we obtain still contains the reproducing kernels for the spherical monogenics. In Sections 2 and 3 we extend the definition of the Fischer product to the setting of monogenic tensors and forms (see also $[8,9]$ ).

\section{The Fischer product for spherical monogenics}

Let $\mathcal{P}_{k}$ denote the right module of $R_{m}$-valued homogeneous polynomials of degree $k$. Then the Fischer inner product on $\mathcal{P}_{k}$ is given by

$$
\left(R_{k}(x), S_{k}(x)\right)=\frac{1}{k !} \bar{R}_{k}\left(\partial_{x}\right) S_{k}(x)
$$

Hereby the map $R_{k}(x) \rightarrow R_{k}\left(\partial_{x}\right)$ stands for replacing the $x_{j}$-variables by partial derivatives and the map $a \rightarrow \bar{a}$ is the main anti-involution on $R_{m}$ determined by $\overline{a b}=\bar{b} \bar{a}$ and $\bar{e}_{j}=-e_{j}$. The above inner product is compatible.with the right module structure and the scalar part of it determines a positive definite inner product on the space of all homogeneous polynomials (in fact on the space of all Clifford polynomials). Hence we may consider the subspace $x \mathcal{P}_{k-1}$ of $\mathcal{P}_{k}$ of polynomials of the form $x R_{k-1}(x)$ and the orthogonal complement of this subspace is clearly the space $\mathcal{M}_{k}$ of spherical monogenics of degree $k$, i.e. of homogeneous polynomials $P_{k}$ satisfying $\partial_{x} P_{k}(x)=0$. This means that every homogeneous Clifford polynomial $R_{k}$ admits a unique orthogonal decomposition of the form

$$
R_{k}(x)=P_{k}(x)+x R_{k-1}(x) \quad\left(R_{k-1} \in \mathcal{P}_{k-1}, \partial_{x} P_{k}(x)=0\right)
$$


called Fischer decomposition and by recursive application of this decomposition one arrives at the complete Fischer decomposition

$$
R_{k}(x)=\sum_{s} x^{s} P_{k-s}(x) \quad\left(P_{k-s} \in \mathcal{M}_{k-s}\right) .
$$

This orthogonal decomposition refines the "harmonic decomposition" of polynomials and in principle there is nothing in the way of defining a "Fischer product" for spherical monogenics in much the same way as for spherical harmonics. Indeed, given two spherical monogenics $P_{k} \in \mathcal{M}_{k}$ and $P_{l} \in \mathcal{M}_{l}$ one may consider the product of polynomials $R_{k+l}=P_{k} P_{l} \in \mathcal{P}_{k+l}$ and then define the "Fischer product" $P_{k} \circ P_{l}$ to be the highest piece in the Fischer decomposition of the polynomials $R_{k+1}$. But again this product conflicts with the right module structure for spherical monogenics because again one may consider products of monogenic polynomials of the form $\left(P_{k}(x) e_{1}\right) P_{l}(x)=P_{k}(x)\left(e_{1} P_{l}(x)\right)$ but the polynomial $e_{1} P_{l}$ is no longer spherical monogenic; it is only spherical harmonic.

This already suggest that the Fischer product for spherical harmonics may even be more natural than that for spherical monogenics and this is indeed true because the Fischer product of spherical harmonics can be defined on the abstract level of ring theory. To that end, let $R$ be a ring and let $I$ be a two-sided ideal in $R$. Then. the quotient space $R / I$ inherits the ring structure from $R$ and the product is given by $(a+I)(b+I)=a b+I$.

In case where $R=\mathcal{P}, \mathcal{P}$ being the ring of all polynomials, we may consider the subspace of polynomials of the form $I=|x|^{2} \mathcal{P}$ which is clearly a two-sided ideal. Moreover, the quotient space $R / I$ is canonically isomorphic to the space of all harmonic polynomials. What goes wrong in Clifford analysis is that the space of Clifford polynomials $I=x \mathcal{P}$, is not a two-sided ideal of the ring $\mathcal{P}$ of all Clifford polynomials; it is only a right ideal. However, it is possible to define a subring $\mathcal{P} b$ of $\mathcal{P}$ for which $I=x \mathcal{P}$ becomes a two-sided ideal.

Definition 1. $\mathcal{P} b$ is the ring of all $R_{\mathrm{m}}$-valued polynomials $R$ which are intertwinable with the polynomial $x=\sum_{j} x_{j} e_{j}$, i.e. there exists a polynomial $S$ for which $x R(x)=$ $S(x) x$.

It is readily seen that in case $x R(x)=S(x) x$ we also have $x S(x) x=x^{2} R(x)=$ $R(x) x^{2}$ and, as $f(x)=x$ is invertible outside the origin, we also have $x S(x)=R(x) x$ so that $R$ and $S$ both belong to $\mathcal{P}$ b. Hence, intertwining with $x$ from the left implies intertwining with $x$ from the right; there is no left - right distinction in Definition 1.

\section{Examples.}

(1) The polynomial $e_{1}$ does not belong to the space $\mathcal{P b}$ for $x e_{1}$ can never be written as $a x$ for some Clifford number $a$.

(2) Any scalar polynomial belongs to $\mathcal{P} b$.

(3) The wedge products $x \wedge u_{1} \wedge \ldots \wedge u_{k}$ belong to $\mathcal{P}$ b because

$$
x\left(x \wedge u_{1} \wedge \ldots \wedge u_{k}\right)=(-1)^{k}\left(x \wedge u_{1} \wedge \ldots \wedge u_{k}\right) x .
$$

(4) The zonal spherical monogenics $Z_{k}(x, u)$ may be defined as the reproducing kernels for the spaces of spherical monogenics $\mathcal{M}_{k}$, i.e. $Z_{k}\left(x, \partial_{u}\right) R_{k}(u)=P_{k}(x)$ in case $R_{k}(x)=P_{k}(x)+\dot{x} R_{k-1}(x) \quad\left(P_{k} \in \mathcal{M}_{k}\right)$ (see also [10]). As they are of the form $A(x, u)+x \wedge u B(x, u)$ for scalar polynomials $A$ and $B$ they belong to $\mathcal{P} b$. 
Lemma 1. The space of Clifford polynomials of the form $x \mathcal{P}$ is a two-sided ideal inside $\mathcal{P b}$.

Proof. Note that any polynomial of the form $x R(x)$ satisfies the intertwining relation $x(x R(x))=(R(x) x) x$ and hence belongs to $\mathcal{P}$ b. That $x \mathcal{P}$ is a right ideal is clear while for any $R \in \mathcal{P}$ b with $x R(x)=S(x) x$ we also have that $x S(x)=R(x) x$ so that $R(x) x \mathcal{P}=x S(x) \mathcal{P}$

It follows from the above lemma that the quotient space $\mathcal{P b} / x \mathcal{P}$ has a natural ring structure inherited from $\mathcal{P}$ b. Moreover, we have the following

Lemma 2. The space $P b / x P$ is canonically isomorphic to the space of monogenic polynomials $P \in \mathcal{P} b$, i.e. $P \in \mathcal{P} b$ and $\partial_{x} P(x)=0$.

Proof. The Fischer inner product remains positive definite after restriction to the spaces $\mathcal{P} b_{k}$ of homogeneous polynomials of degree $k$ in $\mathcal{P}$ b. Hence $\mathcal{P} b_{k} / x \mathcal{P}_{k-1}$ is canonically isomorphic to the orthogonal complement of $x \mathcal{P}_{k-1}$ inside $\mathcal{P} b_{k}$ which is the space of polynomials $P_{k} \in \mathcal{P} b_{k}$ for which $\left[\bar{R}_{k-1}\left(\partial_{x}\right) \partial_{x} P_{k}(x)\right]_{0}=0$ for all $R_{k-1} \in P_{k-1}$, where $[a]_{0}$ denotes the scalar part of $a \in R_{m}$. This means that $P_{k} \in \mathcal{P} b_{k}$ and $\partial_{x} P_{k}(x)=0$

Hence there is a canonical product for monogenic polynomials in $\mathcal{P}$ b.

We conclude this section with the following two remarks:

1. First of all we have to verify that the Fischer product indeed generalizes the product of holomorphic polynomials in the plane $z^{k} z^{l}=z^{(k+l)} \quad(z=x+i y)$. To that end we interprete the complex number $i$ as the bivector $-e_{1} e_{2}$ so that $\left(x-e_{1} e_{2} y\right)^{k}$ is indeed a solution of the Dirac equation

$$
\left(e_{1} \partial_{x_{1}}+e_{2} \partial_{x_{2}}\right) f\left(x_{1}, x_{2}\right)=e_{1}\left(\partial_{x_{1}}+i \partial_{x_{2}}\right) f\left(x_{1}, x_{2}\right)=0
$$

Of course, the pointwise product $z^{k} z^{l}$ is also a Fischer product because the result $z^{(k+l)}$ is monogenic; no further monogenic projection is needed. Finally, the variable $x-e_{1} e_{2} y$ satisfies

$$
\left(x e_{1}+y e_{2}\right)\left(x-e_{1} e_{2} y\right)=\left(x+e_{1} e_{2} y\right)\left(x e_{1}+y e_{2}\right)
$$

so that it intertwines with the vector variable $x e_{1}+y e_{2}$, i.e. it belongs to the class $\mathcal{P} b$ and so does any complex number $a+i b$. The Fischer product in the quotient ring $\mathcal{P} b / x \mathcal{P}$ hence fully generalizes the product of holomorphic functions.

2. Yet the class of polynomials $\mathcal{P} b$ is not the only class for which the Fischer product can be meaningfully defined: it can in principle be defined for all Clifford polynomials, only it is in most cases not very natural to consider this product. But worse than this, the class of polynomials $\mathcal{P b}$ is not the only class for which the Fischer product is naturally defined. Indeed, let $m=2 n$ or $m=2 n+1$. Then for any holomorphic function $f\left(x_{1}+i x_{2}, \ldots, x_{2 n-1}+i x_{2 n}\right)$ the function $I f(x)$, with $I=\left(1+i e_{1} e_{2}\right) \cdots\left(1+i e_{2 n-1} e_{2 n}\right)$ satisfies the Dirac equation. Conversely, if for a scalar function $f, I f$ satisfies the Dirac equation, then $f$ is holomorphic of several complex variables. Moreover, in case $f$ and $g$ are scalar holomorphic functions of several complex variables, then we have that If $I g=I f g$, i.e. the pointwise product of holomorphic functions is another natural example of a Fischer product. We may thus conclude that we have two complementary examples of natural Fischer products. 


\section{The Fischer product. defined for monogenic tensors}

A Clifford $k$-tensor is simply a multilinear function $F\left(u_{1}, \ldots, u_{k}\right)$ of $k$ different vector variables $u_{j}=\sum_{l} u_{j_{l}} e_{l}$ (see also [4]). One can extend Clifford tensors as to include covariant and contravariant tensors or one can also consider Clifford tensors of several variables (bitensors) as in [8], but for this paper the above definition is sufficient. A $k$ tensor $M\left(u_{1}, \ldots, u_{k}\right)$ is called (left) monogenic if it is monogenic in each vector variable, i.e. if

$$
\partial_{u_{j}} M\left(u_{1}, \ldots, u_{k}\right)=0 \quad\left(\partial_{u_{j}}=\sum \partial_{u_{i_{l}}} e_{l}(j=1, \ldots, k)\right)
$$

(see also [9]). Next, let $\mathcal{T}_{k}$ be the right $R_{m}$-module of Clifford tensors $F\left(u_{1}, \ldots, u_{k}\right)$. Then the Fischer inner product on $\mathcal{T}_{k}$ is given by

$$
(G, F)=\bar{G}\left(\partial_{u_{1}}, \ldots, \partial_{u_{k}}\right) F\left(u_{1}, \ldots, u_{k}\right),
$$

like for Clifford polynomials. Moreover, the scalar part $[(G, F)]_{0}$ is positive definite on the space of Clifford tensors and with respect to this inner product every Clifford tensor admits a unique orthogonal decomposition of the form (the monogenic decomposition)

$$
F\left(u_{1}, \ldots, u_{k}\right)=M(F)\left(u_{1}, \ldots, u_{k}\right)+\sum_{j=1}^{k} u_{j} F_{j}\left(u_{1}, \ldots u_{j-1}, u_{j+1}, \ldots, u_{k}\right)
$$

whercby $M(F)\left(u_{1}, \ldots, u_{k}\right)$ is a monogenic tensor.

The importance of this decomposition is that it generalized the Fischer decomposition (monogenic decomposition) for homogeneous Clifford polynomials $R_{k}(u)$ of one vector variable, for homogeneous Clifford polynomials of several vector variables $R_{k_{1} \cdots k_{l}}\left(u_{1}, \ldots, u_{l}\right)$ for Clifford algebra-valued differential forms (see also [2]) as well as for lots of other "tensor types" coming from the application of suitable symmetrization and anti-symmetrization operators, which as we pointed out in [9], commute with the monogenic decomposition. In particular, by using Young symmetry operators together with the monogenic decomposition, one arrives at models for all irreducible finite-dimensional representations of $\operatorname{Spin}(m)$. All this clearly stresses the importance of monogenic tensors and it would be more than important to have a good product for monogenic tensors which would be at the basis of possible products for monogenic polynomials of one and several vector variables as well as for monogenic differential forms. The fundamental product to start from is the product of Clifford tensors $F \in \mathcal{T}_{k}$ and $G \in \mathcal{T}_{l}$ given by

$$
F G\left(u_{1}, \ldots, u_{k+1}\right)=F\left(u_{1}, \ldots, u_{k}\right) G\left(u_{k+1}, \ldots, u_{k+1}\right) .
$$

This product does not disturb possible symmetry properties of the individual tensors $F$ and $G$ and it is hence at the basis of the definition of products for all special Clifford tensor types (i.e. polynomials, differential forms, etc.). Moreover, for this product the direct sum $\mathcal{T}=R_{m}^{\prime}+\mathcal{T}_{1}+\mathcal{T}_{2}+\ldots$ of tensor spaces becomes a ring. Moreover, the subspace $M(\mathcal{T})=R_{m}+M\left(\mathcal{T}_{1}\right)+M\left(\mathcal{T}_{2}\right)+\ldots$ of monogenic tensors is a right $R_{m^{-}}$ submodule of $\mathcal{T}$ and already at this point one could define the Fischer product for 
monogenic tensors $M \in M(\mathcal{T})$ and $M^{\prime} \in M(\mathcal{T})$ to be the monogenic piece $M\left(M M^{\prime}\right)$ in the Fischer decomposition of the tensor product $M M^{\prime}$ of $M$ and $M^{\prime}$. But again this product is incompatible with the right module structure of $M(\mathcal{T})$ again because $M e_{1} \in M(\mathcal{T})$ and $\left(M e_{1}\right) M^{\prime}=M\left(e_{1} M^{\prime}\right)$ while $e_{1} M^{\prime}$ is no longer monogenic. Like in the previous section we have to construct a suitable restriction $\mathcal{T}$ b of the ring $\mathcal{T}$.

Definition 2. $T b_{k}$ is the space of all Clifford tensors $F\left(u_{1}, \ldots, u_{k}\right)$ such that for any extra vector variable $u_{k+1}$, the tensor product $F\left(u_{1}, \ldots, u_{k}\right) u_{k+1}$ has the form

$$
\sum_{j=1}^{k+1} u_{j} F_{j}\left(u_{1}, \ldots, u_{j-1}, u_{j+1}, \ldots, u_{k+1}\right) .
$$

By $\mathcal{T} b$ we denote the direct sum of all spaces $\mathcal{T} b_{k}$.

Examples.

(1) The tensor $e_{1} \in R_{m}$ does not belong to $\mathcal{T}$ b for $e_{1} u, u=\sum e_{j} u_{j}$ can never be written in the form $u a$ with $a \in R_{m}$.

(2) Any scalar-valued tensor belongs to $\mathcal{T}_{b}$ and in particular also the bitensor $K\left(u_{1}, \ldots, u_{k} ; v_{1}, \ldots, v_{k}\right)=\left\langle u_{1}, v_{1}\right\rangle \cdots\left\langle u_{k}, v_{k}\right\rangle$ with $\langle x, y\rangle=\sum x_{j} y_{j}$, which is the reproducing kernel of $\mathcal{T}_{k}$ for the Fischer inner product.

It is left as an exercise to the reader to show that $\mathcal{T} b$ is a subring of the ring of Clifford tensors $\mathcal{T}$, i.e. the tensor product $F G$ of $F \in \mathcal{T}$ b and $G \in \mathcal{T}$ b still belongs to $\mathcal{T}$ b. Next consider for each $k \in \mathbb{N}$ the orthogonal complement $M^{\#}\left(\mathcal{T}_{k}\right)$ of the subspace $M\left(\mathcal{T}_{k}\right)$ of $\mathcal{T}_{k}$ for the Fischer inner product, i.e. the space of Clifford tensors of the form

$$
\sum_{j=1}^{k} u_{j} F_{j}\left(u_{1}, \ldots, u_{j-1}, u_{j+1}, \ldots, u_{k}\right)
$$

Then we may consider the direct sum $M^{\#}(\mathcal{T})$ of these right $R_{m}$-modules $M^{\#}\left(\mathcal{T}_{k}\right)$ and it is clear that $M^{\#}(\mathcal{T})$ is even a right idcal of the ring $\mathcal{T}$ in the same way as $x \mathcal{P}$ is a right ideal of $\mathcal{P}$. But again $M^{\#}(\mathcal{T})$ is not a two-sided ideal of $\mathcal{T}$ which is the obstruction for a natural Fischer product for all Clifford tensors. However, we have

Lemma 3. The ring $M^{\#}(\mathcal{T})$ is a two-sided ideal of the ring $\mathcal{T}$ b.

Proof. It is easy to see that for any tensor $F\left(u_{1}, \ldots, u_{k}\right)$ of the form

$$
\sum u_{j} F_{j}\left(u_{1}, \ldots, u_{j-1}, u_{j+1}, \ldots, u_{k}\right)
$$

the product $F\left(u_{1}, \ldots, u_{k}\right) u_{k+1}$ is of the form

$$
\sum_{j=1}^{k} u_{j} G_{j}\left(u_{1}, \ldots, u_{j-1}, u_{j+1}, \ldots, u_{k+1}\right)
$$

and hence of the special form required for elements of $\mathcal{T}$ b, i.e. $M^{\#}(\mathcal{T})$ is a subspace of $\mathcal{T}$ b and hence also a right ideal: That $M^{\#}(\mathcal{T})$ is also a left ideal of $\mathcal{T}$ b follows from Definition 
2 which implies that the tensor product $F G$ of $F \in \mathcal{T} b_{k}$ and $G=\sum u_{j} G_{j} \in M^{\#}\left(\mathcal{T}_{l}\right)$ is most certainly of the form

$$
F G=\sum_{j=1}^{k+l} u_{j} H_{j}\left(u_{1}, \ldots, u_{j-1}, u_{j+1}, \ldots, u_{k+1}\right)
$$

so that $F G$ belongs to $M^{\#}\left(T_{k+1}\right)$

Corollary. The monogenic piece $M(F)$ of any scalar-valued tensor belongs to $\mathcal{T} b$. In particular, the reproducing kernel for the space of monogenic $k$-tensors $M\left(\mathcal{T}_{k}\right)$, which is the monogenic projection $M\left(u_{1}, \ldots, u_{k} ; v_{1}, \ldots, v_{k}\right)=M\left(\left\langle u_{1}, v_{1}\right\rangle \ldots\left\langle u_{k}, v_{k}\right\rangle\right)$ of the scalar bitensor $K\left(u_{1}, \ldots, u_{k} ; v_{1}, \ldots, v_{k}\right)=\left\langle u_{1}, v_{1}\right\rangle \ldots\left\langle u_{k}, v_{k}\right\rangle$ belongs to $\mathcal{T}$ b.

Proof. For any scalar tensor $F \in \mathcal{T}_{k}$ the monogenic projection $M(\mathcal{T})$ is a sum of the form $F+\sum u_{j} F_{j}\left(u_{1}, \ldots, u_{j-1}, u_{j+1}, \ldots, u_{k}\right)$ for suitable tensors $F_{j}$, and by Lemma 3 , this clearly belongs to $\mathcal{T} b$

Hence the most fundamental monogenic tensor belongs to $\mathcal{T}$ b. Due to Lemma 2, the quotient space $\mathcal{T} b / M^{\#}(\mathcal{T})$ has a natural ring structure whereby the product is abstractly defined by the formula $\left(F+M^{\#}(\mathcal{T})\right)\left(G+M^{\#}(\mathcal{T})\right)=F G+M^{\#}(\mathcal{T})$. To complete the discussion we still prove the following

Lemma 4. The quotient space $T b / M^{\#}(\mathcal{T})$ is naturally isomorphic to the space of monogenic tensors belonging to $\mathcal{T}$ b, i.e. $\mathcal{T} b \cap M(\mathcal{T})$, which is also the monogenic projection $M(\mathcal{T} b)$ of $\mathcal{T}$ b.

Proof. On the one hand, similar to Lemma 3 one can prove that the monogenic projection $M(F)$ of $F \in \mathcal{T}$ b still belongs to $\mathcal{T}$ b. On the other hand, the Fischer inner product remains positive definite after restriction to $\mathcal{T}$ b so that in fact $\mathcal{T} b / M^{\#}(\mathcal{T})$ is a vector space isomorphic to the or thogonal complement of $M^{\#}(\mathcal{T})$ inside $\mathcal{T} b$ with respect to the Fischer inner product, which is the intersection $\mathcal{T} b \cap M(\mathcal{T})$

Note that hence the monogenic projection $F \rightarrow M(F)(F \in \mathcal{T}$ b) drops to the isomorphism $F+M^{\#}(\mathcal{T}) \rightarrow M(F)$ between the quotient ring $\mathcal{T} b / M^{\#}(\mathcal{T})$ and the space $M(\mathcal{T} b)$ and under this isomorphism the product in the quotient $\operatorname{ring} \mathcal{T} b / M^{\#}(\mathcal{T})$ corresponds to the binary operation

$$
(M(F), M(G)) \rightarrow M(M(F) M(G)) \quad(F, G \in \mathcal{T} \text { b) }
$$

For example, denote by $M_{k}$ the reproducing kernel for the space of monogenic $k$-tensors: $M_{k}=M\left(\left\langle u_{1}, v_{1}\right\rangle \ldots\left\langle u_{k}, v_{k}\right\rangle\right)$. Then it is readily verified that the Fischer product of $M_{k}$ and $M_{l}$ is just the reproducing kernel $M_{k+l}$ of $M\left(\mathcal{T}_{k+l}\right)$. In other words, the Fischer product reduces to a mere shift in the index of the reproducing kernel.

Note that the class $\mathcal{T} b$ is in fact the maximal class of tensors for which $M^{\#}(\mathcal{T})$ is a two-sided ideal; the condition to be satisfied by the elements of $\mathcal{T}$ b indeed expresses the fact that the tensor product $F G$ of $F \in \mathcal{T}$ b and $G \in M^{\#}(\mathcal{T})$ again belongs to $M^{\#}(\mathcal{T})$. In the same way, the class of polynomials $\mathcal{P} b$ was defined as the maximal class 
of polynomials for which $x \mathcal{P}$ is a two-sided ideal. Hence one may wonder that, under the process of total symmetrization

$$
F\left(u_{1}, \ldots, u_{k}\right) \rightarrow \frac{1}{k !} \sum_{\pi} F\left(u_{\pi(1)}, \ldots, u_{\pi(k)}\right)
$$

and after identifying totally symmetric tensors with polynomials via the isomorphism $F\left(u_{1}, \ldots, u_{k}\right) \rightarrow F(u, \ldots, u)$ the class of tensors $\mathcal{T}$ b projects onto $\mathcal{P}$ b. To answer this, first note that the above map $\mathcal{T} \rightarrow \mathcal{P}$ is simply given by the replacement $F\left(u_{1}, \ldots, u_{k}\right) \rightarrow$ $F(u, \ldots, u)$. Hence in case $F\left(u_{1}, \ldots, u_{k}\right)$ satisfies the condition for $\mathcal{T}$ b

$$
F\left(u_{1}, \ldots, u_{k}\right) u_{k+1}=\sum_{j=1}^{k+1} u_{j} F_{j}\left(u_{1}, \ldots, u_{j-1}, u_{j+1}, \ldots, u_{k+1}\right)
$$

then the polynomial $F(u, \ldots, u)$ satisfies

$$
F(u, \ldots, u) u=u \sum_{j=1}^{k+1} F_{j}(u, \ldots, u)
$$

and therefore belongs to $\mathcal{P} b$, i.e. $\mathcal{T}$ b projects to a subring of $\mathcal{P} b$. But $\mathcal{T} b$ projects to a proper subring of $\mathcal{P} b$ because the linear function $x \rightarrow x \wedge u \wedge v$ belongs to $\mathcal{P} b$ and can at the same time be shown not to belong to $\mathcal{T}$ b, i.e. the product $(x \wedge u \wedge v) y$ is not of the form $x F(y)+y G(x)$ for certain linear functions $F$ and $G$. At first this may look surprising because tensor calculus is really at the basis of polynomial and differential form calculus. But on the other hand, the tensor product, even for symmetric tensors, is much more than just the tensor formulation of a polynomial product. For example, a linear function $F$ may be interpreted both as polynomial and as a one-form, but the polynomial product of linear functions is quite different from the exterior product of one-forms. The tensor product of linear functions includes both products in the sense that polynomial and exterior product are expressible in terms of the tensor product and the class $\mathcal{T}$ b was defined for the tensor product. We finish this section with the complete characterization of the class of tensors $\mathcal{T} b$ in the following

Theorem 1. Any tensor $F \in \mathcal{T}$ b may be written (not in a unique way) as a sum

$$
F(u)=F_{s}(u)+\sum u, F_{j}\left(u_{1}, \ldots, u_{j-1}, u_{j+1}, \ldots, u_{k}\right)
$$

of a tensor of the form $F_{s}(u)=G_{s}(u)+e_{1} \ldots e_{m} H_{s}(u)$ with $G_{s}, H_{s}$ scalar tensors and a tensor belonging to the class $M^{\#}(\mathcal{T})$.

Proof. For tensors of length zero, i.e. $F \in \mathcal{T} b_{0}=R_{m}$ the condition of Definition 2 simply means that $F$ satisfies the intertwining relation $F u=u G$ for some $G \in R_{m}$ from which it follow that, for all $j=1, \ldots, m, F=-e_{j} G e_{j}$ and hence also that $e_{j} G e_{j}=e_{k} G e_{k}$. From this last condition it follows that $G$ has the form "scalar $+e_{1} \cdots e_{m}$ scalar", and as the pseudoscalar commutes or anticommutes with all $e_{j}$ 's, it follows that $F=$ scalar $+e_{1} \cdots e_{m}$ scalar. 
The general property can be proved by induction on the length of the tensor. As it is rather complicated we suffice by proving the result for tensors of length one, i.e. for linear function $F(u)=\sum F_{j} u_{j} \quad\left(F_{j} \in R_{m}\right)$. The condition $F(u) v=u G(v)+v H(u)$ from Definition 2 may be rewritten as a system of equations for Clifford numbers $F_{j} e_{k}=$ $e_{j} G_{k}+e_{k} H_{j}$ from which we obtain that $e_{j} F_{j}=G_{k} e_{k}+e_{j} e_{k} H_{j} e_{k}$. Hence we also obtain that $e_{i} F_{i}-e_{j} F_{j}=e_{i} e_{k} H_{i} e_{k}-e_{j} e_{k} H_{j} e_{k}$ which leads to the relations for the elements $H_{j} \in R_{m}$

$$
e_{i} e_{k} H_{i} e_{k}-e_{j} e_{k} H_{j} e_{k}=i b i d(k \rightarrow l)
$$

from which the Theorem is to be obtained.

First let us assume these last relations for all $k \neq i, j$. Then $e_{k}\left(e_{i} H_{i}-e_{j} H_{j}\right) e_{k}=$ $i b i d(k \rightarrow l)$ from which it readily follows that the expression $e_{i} H_{i}-e_{j} H_{j}$ has the form

$$
A_{i j}+e_{i} B_{i j}+e_{j} C_{i j}+e_{i j} D_{i j}+e_{1} \ldots e_{m} \text { dito }
$$

whereby $A_{i j}, B_{i j}, C_{i j}$ and $D_{i j}$ are scalars. Next we can writc down the identity for $k=i$ and any $l \neq i, j$

$$
-H_{i} e_{i}-e_{j} e_{i} H_{j} e_{i}=A_{i j}-e_{i} B_{i j}-e_{j} C_{i j}+e_{i j} D_{i j}+e_{1} \ldots e_{\text {rn }} \text { dito. }
$$

Hence the expressions $H_{i}+e_{i} e_{j} H_{j}$ and $H_{i}+e_{j} e_{i} H_{j}$ are given by

$$
\left.\begin{array}{l}
H_{i}+e_{i} e_{j} H_{j}=-e_{j} A_{i j}+B_{i j}-e_{i j} C_{i j}+e_{j} D_{i j}+e_{1} \cdots e_{m} \text { dito } \\
H_{i}+e_{j} e_{i} H_{j}=e_{i} A_{i j}+B_{i j}+e_{i j} C_{i j}+e_{j} D_{i j}+e_{1} \cdots e_{m} \text { dito }
\end{array}\right\}
$$

so that, taking the sum, $H_{i}=B_{i j}+e_{j} D_{i j}+e_{1} \cdots e_{m}$ dito. Moreover, as this is true for all $j$, we must have that $H_{i}=B_{i}+e_{1} \cdots e_{m}$ dito $\left(B_{i} \in R\right)$. Hence we arrive at $F_{j}=$ $-e_{j} G_{k} e_{k}+B_{j}+e_{1} \cdots e_{m}$ dito so that also (take, e.g., $\left.k=1\right) F(u)=u G^{\prime}(u)+\sum B_{j} u_{j}$

The previous theorem in fact characterizes the monogenic product for tensors completely because the quotient $\operatorname{ring} \mathcal{T} b / M^{\#}(\mathcal{T})$ is clearly isomorphic to the space $M\left(\mathcal{T}_{s}+\right.$ $\left.e_{1} \cdots e_{m} \mathcal{T}_{s}\right)$ of monogenic projections of scalar and pseudoscalar tensors. This space is spanned (over the set of numbers of the form $a+e_{1} \cdots e_{m} b \quad(a, b \in R)$ ) by the collection of fundamental tensors $M\left(u_{j_{1}} \cdots u_{j_{k}}\right)=M\left(\left\langle u, e_{j_{1}}\right\rangle \ldots\left\langle u, e_{j_{k}}\right\rangle\right)$ for which we have already evaluated the Fischer product; it was merely a matter of shifting the indices. We may thus conclude that we have fully evaluated the product in the $\operatorname{ring} \mathcal{T} b / M^{\#}(\mathcal{T})$ and as $\mathcal{T}$ b was in fact defined as the largest ring of tensors for which the above quotient is still a ring, we have come to the end of a certain line of thinking. 


\section{Products for special tensor types}

Polynomials of one or several vector variables and Clifford differential forms are basic ingredients of what one could call the "Clifford analysis toolkit", i.e. they are natural objects to work with from a calculus point of view. The algebra of these objects is generated by

(i) the basic Clifford numbers $e_{1}, \ldots, e_{m}$ generating the Clifford algebra $R_{m}$

(ii) any number of vector variables $u_{j}=\sum u_{j_{k}} e_{k}$ whereby the coordinates $u_{j_{k}}$ form a polynomial algebra

(iii) any number of vector differentials $d u_{j}=\sum d u_{j_{k}} e_{k}$ whereby the elements $d u_{j_{k}}$ multiply like generators of a Grassmann algebra.

An element of this algebra is a Clifford differential form with polynomial coefficients and algebras of Clifford differential forms were studied to some extent in $[3,7$, 10]. From the above, it is clear how the multiplication of Clifford differential forms with polynomial coefficient $F\left(u_{1}, \ldots, u_{k} ; d u_{1}, \ldots, d u_{k}\right)$ works; it is simply a mixture of Clifford multiplication, polynomial multiplication and differential form multiplication.

Of course, the basic toolkit for Clifford analysis is not complete with the introduction of this algebra; one also needs various endomorphisms on this algebra of which we mention the following ones:

(i) Dirac operators (or vector derivatives) $\partial_{u_{j}}=\sum \partial_{u_{j_{k}}} e_{k}$ which may act from the left or from the right on Clifford differential forms: $\partial_{u_{j}} F=\sum e_{k} \partial_{u_{j_{k}}} F$ and $F \partial_{u_{j}}=$ $\sum \partial_{u_{j_{k}}} F e_{k}$ $F e_{k}$

(ii) Left and right contraction operators $\partial_{u_{j}}\left|F=\sum e_{k} \partial_{u_{j_{k}}}\right| F$ and $F\left|\partial_{u_{j}}=\sum \partial_{u_{j_{k}}}\right|$

(iii) The exterior derivative $d: F \rightarrow d F$ which is the sum $d=d_{u_{1}}+\ldots+d_{u_{k}}$ of all exterior derivatives $d_{u_{j}}=\sum d u_{j_{k}} \partial_{u_{j_{k}}}$ with respect to all vector variables $u_{1}, \ldots, u_{k}$ under consideration.

(iv) Dual to the exterior derivative $d$, which transforms commuting variables $u_{j_{k}}$ into anti-commuting variables $d u_{j_{k}}$, one could even consider something like an operator "|" transforming commuting derivatives $\partial_{u_{i_{k}}}$ into anti-commuting derivatives $\partial_{u_{i_{k}}} \mid$, which are the basic contraction operators. Note hereby that the contraction operators $\partial_{u_{j_{k}}} \mid$ indeed behave like anti-commuting derivatives; they are determined by $\partial_{u_{j_{k}}} \mid d u_{l_{n}}=\delta_{j_{1}} \delta_{k_{n}}$ together with the product rule

$$
\partial_{u_{j_{k}}} \mid(F G)=\left(\partial_{u_{j_{k}}} \mid F\right) G+(-1)^{k} F\left(\partial_{u_{j_{k}}} \mid G\right)
$$

$F$ being a scalar $k$-form.

The formal calculus thus obtained is sclf-consistent and its rules are clear without any further need to relate things to Clifford tensors in order to give a mathematical explanation for differential forms. Everything follows from the axiomatic calculus of commuting and anti-commuting variables together with Clifford numbers, also the 
integration of differential forms along oriented surfaces. Indeed, let $F(x, d x) \quad(x=$ $\left.\sum e_{j} x_{j}, d x=\sum d x_{j} e_{j}\right)$ be a Clifford $k$-form and consider a piece of oriented surface given by a mapping

$$
\left(u_{1}, \ldots, u_{k}\right) \in O \subset R^{k} \rightarrow x\left(u_{1}, \ldots, u_{k}\right)
$$

Then one applies the chain rule $d x\left(u_{1}, \ldots, u_{k}\right)=\sum \frac{\partial x_{j}}{\partial u_{l}} e_{j} d u_{l}$ to arrive at a differential form $F(x(u), d x(u))=G(u) d u_{1} \cdots d u_{k}$ which can be integrated over the domain $O$ the result being the integral of $F$ over the surface $x(O)$. Note that the oriented surface measure on $x(O)$ corresponds to the differential form $d x^{k}$.

We now define a conjugation for polynomial Clifford forms as follows:

(i) For $a \in R_{m}$ the conjugate is given by the main anti-involution $a \rightarrow \bar{a}$, i.e. $\bar{e}_{j}=-e_{j}$ and $\overline{a b}=b a$.

(ii) For a scalar variable $u_{j_{k}}$, the conjugate is the derivative $\partial_{u_{j_{k}}}$ with respect to that variable.

(iii) The conjugate of a differential $d u_{j_{k}}$ is the contraction operator $\partial_{u_{j_{k}}} \mid$ corresponding to that differential.

(iv) The conjugation is denoted formally as a map

$$
+: F\left(u_{1}, \ldots, u_{n} ; d u_{1}, \ldots, d u_{n}\right) \rightarrow \tilde{F}\left(\partial_{u_{1}}, \ldots, \partial_{u_{n}} ; \partial_{u_{1}}\left|, \ldots, \partial_{u_{n}}\right|\right)
$$

and behaves like an anti-involution, i.e. $(F G)^{+}=G^{+} F^{+}$. In particular, for a product $d u_{j_{1} l_{1}} \cdots d u_{j_{k} l_{k}}$ the conjugate is the operator of contraction $\partial_{u_{j_{k} l_{k}}}\left|\cdots \partial_{u_{j_{1} l_{1}}}\right|$ (note that the conjugation is well defined in this way and can be extended to an actual antiinvolution in a unique way).

Next we consider polynomial Clifford forms $F\left(u_{1}, \ldots, u_{n} ; d u_{1}, \ldots, d u_{n}\right)$ which are homogeneous of degree $k_{1}, \ldots, k_{n}$ in the variables $u_{1}, \ldots, u_{n}$ and of degree $l_{1}, \ldots, l_{n}$ in the variables $d u_{1}, \ldots, d u_{n}$. Then for two such polynomial Clifford forms, the action

$$
G^{+} F=\bar{G}\left(\partial_{u_{1}}, \ldots, \partial_{u_{n}} ; \partial_{u_{1}}\left|, \ldots, \partial_{u_{n}}\right|\right) F\left(u_{1}, \ldots, u_{n} ; d u_{1}, \ldots, d u_{n}\right)
$$

is a Clifford number leading to the Fischer inner product

$$
(G, F)=\frac{1}{k_{1} !} \cdots \frac{1}{k_{n} !} \frac{1}{l_{1} !} \cdots \frac{1}{l_{n} !} G^{+} F
$$

defined on the right $R_{m}$-module of homogeneous polynomial Clifford forms and hence extendable to the ring of all polynomial Clifford forms $\Phi$. It is easy to see that the scalar part $\{(G, F)]_{0}$ of the Fischer inner product defines a positive definite scalar inner product on each homogeneous subspace $\Phi_{k_{1}, \ldots, k_{n} ; l_{1}, \ldots, l_{n}}$ of the ring $\Phi$ and hence also on the ring $\Phi$ itself.

Next we introduce the monogenic decomposition. First we have the following 
Definition 3. A polynomial Clifford form $F\left(u_{1}, \ldots, u_{n} ; d u_{1}, \ldots, d u_{n}\right)$ is called (left) monogenic if it satisfies the system

$$
\left.\begin{array}{r}
\partial_{u_{j}} F\left(u_{1}, \ldots, u_{n} ; d u_{1}, \ldots, d u_{n}\right)=0 \\
\partial_{u_{j}} \mid F\left(u_{1}, \ldots, u_{n} ; d u_{1}, \ldots, d u_{n}\right)=0
\end{array}\right\} \quad(j=1, \ldots, n) .
$$

The subspace of monogenic elements in $\Phi$ is denoted by $M(\Phi)$.

\section{Remarks.}

(1) From the previous definition it is clear what are monogenic forms $F\left(u_{1}, \ldots, u_{n}\right.$; $\left.d u_{1}, \ldots, d u_{n}\right)$ in general (not only polynomial ones) - they are the solutions of $\partial_{u_{j}} F=$ $\partial_{u_{j}} \mid F=0 \quad(j=1, \ldots, n)$.

(2) From the relation $\partial_{u_{j}}=d \partial_{u_{j}}\left|+\partial_{u_{j}}\right| d$ it follows that for any monogenic form $F$, the exterior derivative $d F$ is still monogenic. In this way one arrives at a "complex of monogenic forms" which generalizes the complex of holomorphic forms $F\left(z_{j}, d z_{j}\right)$ of several complex variables (i.e. the holomorphic de Rham complex, not the Dolbeault complex).

Next consider the space $M^{\#}(\Phi)$ of polynomial Clifford forms of the form

$$
F=\sum u_{j} F_{j}+\sum d u_{j} F_{j}^{\prime} \quad\left(F_{j}, F_{j}^{\prime} \in \Phi\right)
$$

Then from the previous definitions one easily obtains the following

Theorem 2 (Fischer decomposition). Every polynomial Clifford form $F \in \Phi$ admits an orthogonal decomposition of the form $F=M(F)+M^{\#}(F), M(F) \in M(\Phi)$ being the monogenic projection of $F$ and $M^{\#}(F) \in M^{\#}(\Phi)$.

By recursive application of this theorem one arrives at a complete and unique Fischer decomposition which is not orthogonal but which can nevertheless be decomposed into orthogonal pieces :

$$
F=M_{0}(F)+M_{1}(F)+\ldots+M_{k+l}(F)
$$

whereby $M_{0}(F) \in M_{0}(\Phi)=M(\Phi)$ is the monogenic piece and $M_{j}(F) \in M_{j}(\Phi)$ has the form $M_{j}(F)=\sum u_{i} F_{i}+\sum d u_{i} F_{i}^{\prime}\left(F_{i} \in M_{j-1}(\Phi)\right)$. This can be proved directly or obtained from [9] after translating differential forms into tensors. In the definition of $M^{\#}(\Phi)$ and $M_{k}(\Phi)$ it is a little strange that vector variables $u_{j}$ and vector differentials $d u_{j}$ are mixed up. It is possible to separate the vector variables $u_{j}$, and the vector differentials $d u_{j}$ in the above Fischer decomposition. Indeed, one can decompose the spaces $M_{k}(\Phi)=M_{k, 0}(\Phi)+\ldots+M_{k, k}(\Phi)$ whereby $M_{0,0}(\Phi)=M_{0}(\Phi), F \in M_{k, 0}(\Phi)$ has the form $\sum d u_{j} F_{j}$ with $F_{j} \in M_{k-1,0}(\Phi)$ and whereby $F \in M_{k, j}(\Phi)$ has the form $\sum u_{j} F_{j}+\sum d u_{j} G_{j}$ with $F_{j} \in M_{k-1, j-1}(\Phi)$ and $G_{j} \in M_{k-1, j}(\Phi)$.

The above refinement of the Fischer decomposition can be shown to be a direct sum decomposition for $m \geq 3$ (see also [9]). But it is no orthogonal decomposition for $m=2$ not even a direct sum (see also [9: Lemma 1]). For example, we have that

$$
\left.\begin{array}{l}
\left(u_{1} e_{1}+u_{2} e_{2}\right)\left(d u_{1} e_{1}-d u_{2} e_{2}\right) \in M_{1,1}(\Phi) \\
\left(d u_{1} e_{1}+d u_{2} e_{2}\right)\left(u_{1} e_{1}-u_{2} e_{2}\right) \in M_{1,0}(\Phi)
\end{array}\right\}
$$


and both forms are equal.

Note that $d: M_{k, 0}(\Phi) \rightarrow M_{k, 0}(\Phi)$, which leads to a set of new complexes including the above mentioned complex $d: M_{0}(\Phi) \rightarrow M_{0}(\Phi)$. We also have that $d: M_{k, j}(\Phi) \rightarrow M_{k, j}(\Phi)+M_{k, j-1}(\Phi)$, which may give rise to even more complexes, but only $d: M_{k, 0}(\Phi) \rightarrow M_{k, 0}(\Phi)$ is a translation invariant complex defined in domains of $\left(R^{m}\right)^{n}$. Now let us turn to the definition of algebra of monogenic forms. To study these one does not need the complete Fischer decomposition; the statement of Theorem 2 is sufficient. The treatment of the product of monogenic forms is similar to the cases studied before: first we consider a subring $\Phi b$ of $\Phi$ consisting of those elements $F \in \Phi$ satisfying the two (or rather $2 n$ ) conditions

$$
\left.\begin{array}{rl}
F u_{j} & =\sum u_{i} F_{i}+\sum d u_{i} F_{i}^{\prime} \\
F d u_{j} & =\sum u_{i} F_{i}+\sum d u_{i} F_{i}^{\prime}
\end{array}\right\} \quad\left(j=1, \ldots, n ; \text { for some } F_{j}, F_{j}^{\prime} \in \Phi\right) .
$$

Next, it is easily seen that the ring $M^{\#}(\Phi)$ is a two-sided ideal of the ring $\Phi b$ and that, moreover, due to Theorem 2, the quotient ring $\Phi b / M^{\#}(\Phi)$ is canonically isomorphic to the space $M(\Phi b)$ of monogenic projections of elements in $\Phi b$. In this way the Fischer product for monogenic differential forms belonging to $\Phi b$ is canonically defined and $\Phi b$ is the largest subring of $\Phi$ which still contains $M^{\#}(\Phi)$ as a two-sided ideal. Hence again we have come to the end of a certain line of thinking. It is important to remark that the exterior derivative " $d$ " leaves the ring $\Phi b$ invariant due to the fact that $d(F) u_{j}=$ $d\left(F u_{j}\right)-(-1)^{\operatorname{deg} F} F d u_{j} \in M^{\#}(\Phi)$ and $d(F) d u_{j}=d\left(F d u_{j}\right) \in M^{\#}(\Phi)$. Hence $d$ : $M(\Phi b) \rightarrow M(\Phi b)$.

We conclude this section with the following two remarks.

1. The first one has to do with the fact that we defined all operations in this section for differential forms with polynomial coefficients. In analysis and in particular in the treatment of cohomology problems one is more interested in differential forms with general analytic or smooth coefficients, not polynomial ones. But analytic functions can be seen as limits of polynomials and the operations introduced in this section (exterior derivative, products) carry over while taking the limit. This section was only intended to introduce basic tools.

2. There is the "tensor interface". It is clear that a complete theory of Clifford differential forms including integration, cohomology and monogenicity can be introduced in a fully self-consistent way without any need to define differential forms (and polynomials) as alternating (symmetric) tensors. On the other hand the translation of Clifford differential forms $F \in \Phi$ into tensors can be useful. For instance, the Fischer decomposition carries over from tensors to differential forms and no further proof of it is needed. This translation works as follows. Let $F\left(u_{1}, \ldots, u_{n} ; d u_{1}, \ldots, d u_{n}\right) \in \Phi$ be homogeneous of degree $\left(k_{1}, \ldots, k_{n}\right)$ in $\left(u_{1}, \ldots, u_{n}\right)$ and of degree $\left(l_{1}, \ldots, l_{n}\right)$ in $\left(d u_{1}, \ldots, d u_{n}\right)$. Then one can interprete $F$ as a tensor of degree $k+l \quad\left(k=\sum k_{j}, l=\sum l_{j}\right)$ in the variables $\left(v_{1}, \ldots v_{k+l}\right)$ in a unique way such that $F\left(v_{1}, \ldots, v_{k+l}\right)$ is symmetric in the sets of variables $\left(v_{1}, \ldots, v_{k_{1}}\right), \ldots,\left(v_{k-k_{n+1}}, \ldots v_{k}\right)$ and anti-symmetric in the sets of variables $\left(v_{k+1}, \ldots, v_{k+l_{1}}\right), \ldots,\left(v_{k+1-l_{n+1}}, \ldots, v_{k+1}\right)$. Moreover, this representation is the inverse of the projection operator which maps a general $k+l$ tensor $F\left(v_{1}, \ldots, v_{k+l}\right)$ onto a 
differential form $F\left(u_{1}, \ldots, u_{n} ; d u_{1}, \ldots, d u_{n}\right)$ of degree $\left(k_{1}, \ldots, k_{n} ; l_{1}, \ldots, l_{n}\right)$, projection operator which is given by the simple formula

$$
F\left(v_{1}, \ldots, v_{k+1}\right) \rightarrow F\left(u_{1}, \ldots, u_{1} ; \ldots ; u_{n}, \ldots, u_{n} ; d u_{1}, \ldots, d u_{1} ; \ldots ; d u_{n}, \ldots, d u_{n}\right) .
$$

This projection preserves the monogenic decomposition and maps the tensor product to the $\Phi$-product and $\mathcal{T} b$ to a proper subclass of $\Phi b$.

\section{References}

[1] Brackx, F., Delanghe, R. and F. Sommen: Clifford Analysis (Res. Notes Math.: Vol. 76). London: Pitman 1982.

[2] Delanghe, R., Sommen, F. and V. Souček: Clifford algebra and spinor valued functions: a function theory for the Dirac operator (Math. and Its Appl.: Vol. 53). Dordrecht: Kluwer Acad. Publ. 1992.

[3] Dimakis, A. and F. Mueller-Hoissen: Clifform calculus. Class. Quantum Gravity (to appear).

[4] Hestenes, D. and G. Sobczyk: Clifford Algebra to Geometric Calculus. Dordrecht: D. Reidel 1985.

[5] Sommen, F.: A product and an exponential function in hypercomplex function theory. Appl. Anal. 12 (1981), $13-26$.

[6] Sommen, F.: Monogenic functions on surfaces. J. Reine Angew. Math. 361 (1985), 145 -161 .

[7] Sommen, F.: Monogenic differential calculus. Trans. Amer. Math. Soc. 326 (1991), 613 -632 .

[8] Sommen, F.: SO(m)-invariant operators on Clifford tensors. In: Fundamental Theories of Physics: Vol. 55; Proc. Third Conf. Clifford Algebras and Appl. in Math. Phys., Deinze 1993 (eds: F. Brackx, R. Delanghe and H. Serras). Dordrecht: Kluwer Acad. Publ. 1993, pp. $193-202$.

[9] Sommen, F.: Clifford tensor calculus. In: Advances in Applied Clifford Algebras; Proc. XXII DGM-Conf., Ixtapa 1993 (ed.: J. Keller and Z. Oziewicz). (Proc. Suppl.) 4 (S1) (1994), $423-435$.

[10] Sommen, F. and B. Janawiecz: Explicit solutions of the inhomogeneous Dirac equation. J. d'Anal. Math. (to appear).

[11] Sommen, F. and V. Souček: Monogenic Differential Forms. Complex Variables : Theory Appl. 19 (1992), 81 - 90.

[12] Vilenkin, M.: Special Functions and the Theory of Group Representations (Transl. Math. Monogr.: Vol. 2). Providence (R.I.): Amer. Math. Soc. 1968. 